\title{
COMPONERSE CON LA CIUDAD: LOS PASEOS SONOROS DE HILDERGARD WESTERKAMP, CHRISTINA KUBISCH Y JANET CARDIFF
}

\author{
SETTING UP WITH THE CITY: THE SOUND WALKS OF HILDERGARD WESTERKAMP, CHRISTINA KUBISCH \\ AND JANET CARDIFF
}

\section{RESUMEN}

El paseo sonoro es una práctica artística que combina el caminar con la escucha del entorno sonoro que se va recorriendo. En este artículo nos acercamos a tres artistas sonoras que se han convertido en referentes ineludibles en cuanto a los paseos sonoros se refiere para analizar cómo estos pueden afectar y alterar nuestra manera de percibir y de habitar nuestras ciudades: Hildergard Westerkamp, Christina Kubisch y Janet Cardiff. Fue precisamente Westerkamp quien dio nombre a esta práctica del salir a pasear para escuchar. Para ella, el paseo sonoro es una efectiva manera de hacernos salir de nuestras burbujas sonoras y tomar conciencia de nuestra participación y responsabilidad en los entornos sonoros urbanos que habitamos. De ahí que la considere una práctica no sólo artística, sino ecológica. Por su parte, Kubisch invita con sus paseos sonoros eléctricos a sumergirse en el paisaje sonoro compuesto por los campos electromagnéticos generados por la multitud de dispositivos eléctricos que nos rodean. De este modo, haciendo perceptible aquello imperceptible con lo que convivimos, nuestras calles se ven recontextualizadas y nuestros sentidos se vuelven más atentos a cuánto nos puede estar pasando desapercibido. Finalmente, Cardiff trama paseos sonoros en los que la voz, y con ella la palabra, cobran especial protagonismo para conducirnos por laberintos afectivos y temporales a través de unas calles siempre compartidas y pobladas de sonidos, memorias, deseos, temores, historia y relatos. A través del trabajo de estas tres artistas nos acercamos al papel clave que el sonido tiene en nuestras vidas y nuestras ciudades y a cómo al tomarnos el tiempo para caminar y escuchar las calles no sólo las ocupamos, sino que nos componemos con ellas.

Palabras clave: Paseo sonoro. Audio Walk. Escucha. Ecología. Ciudad.

\begin{abstract}
The soundwalk is an artistic practice that combines walking with listening to the sound environment. In this article, we present the work of three sound artists who have become unavoidable references in the field of sound walks: Hildergard Westerkamp, Christina Kubisch and Janet Cardiff. We will analyse with them how the soundwalks can affect and alter our way of perceiving and inhabiting our cities. It was precisely Westerkamp who gave name to this practice of going for a walk to listen. For her, the soundwalk is an effective way to get out of our sound bubbles and become aware
\end{abstract}

Susana Jiménez Carmona

Universitat de Barcelona. E-mail: susanajiemenezcarmona@ub.edu 
of our participation and responsibility in the urban sound environments that we inhabit. Hence, she weighs it not only an artistic practice, but also an ecological one. On her part, Kubisch invites us with her Electric Walks to immerse ourselves in the soundscape of the electromagnetic fields generated by the multitude of electrical devices that surround us. Thus, making the nearby imperceptible perceptible, our streets are recontextualised and our senses become more attentive to how much goes unnoticed. Finally, Cardiff weaves audio walks in which the voice, and with it the word, take on special prominence to lead us through emotional and temporal labyrinths through streets always shared and full of sounds, memories, wishes, fears, history and stories. The work of these three artists shows how the sound plays a key role in our lives and our cities and if we take the time to walk and listen to the streets we are not only occupying them but also composing ourselves with them.

Keywords: Soundwalk. Audio Walk. Listening. Ecology. City.

\section{Introducción: caminar la ciudad}

En Wanderlust. Una historia del caminar escribe Rebecca Solnit que al caminar se provoca y se produce un conocimiento recíproco entre nuestros cuerpos percipientes en movimiento y el mundo que percibimos al irlo recorriendo (SOLNIT, 2015, p. 55). Esta reciprocidad mutua de cuerpos y mundo al caminar complica su trama cuando lo que se transitan son las calles de las ciudades en las que vivimos, esos espacios públicos que no sólo activan nuestros sentidos, sino también memorias, afectos, deseos y vínculos personales y colectivos: "Caminar por las calles vincula la lectura del mapa con la propia vida vivida, el microcosmos personal con el macrocosmos público: caminar permite entender el laberinto alrededor" (SOLNIT, 2015, p. 262). Es por ello que ocupar las calles, estar en el espacio público es a la vez hacerlo propio y hacerse parte de él, algo que evidencian reivindicaciones feministas como "Reclaim the Night" o "La calle y la noche también son nuestras" (tampoco podemos olvidar las reivindicaciones antirracistas que luchan asimismo porque las calles sean lugares de y seguros para cualquiera). Porque quienes son asignadas por mandato de género a los espacios interiores experimentan cotidianamente que las calles no son simples trayectos indiferentes y neutros que hay que recorrer para ir de un interior a otro (de nuestra casa al lugar de trabajo, por ejemplo), sino lugares profundamente marcados socialmente desde los que se construyen y nos construyen las ciudades que habitamos.

En este artículo queremos explorar los entrelazamientos y las travesías entre cuerpos y mundos, microcosmos y macrocosmos, interiores y exteriores que se dan, activan o proponen en los trabajos de tres artistas sonoras que son ineludibles referentes de la práctica artística denominada paseo sonoro. Parafraseando las palabras de Solnit sobre el caminar como arte, podríamos decir que analizaremos cómo los soundwalks de Hildegard Westerkamp (nacida en 1946), los electrical walks de Christina Kubisch (nacida en 1948) y los audio walks de Janet Cardiff (nacida en 
1957) llaman la atención sobre los aspectos más simples y también más complejos del propio acto de caminar: cómo se mide el cuerpo con el entorno sonoro, cómo nos afectan los impredecibles encuentros sociales que ofrecen las calles de una ciudad, cómo el acto de una persona puede invitar a la imaginación de otra, cómo cartografiamos el mundo al caminarlo o cómo nos componemos con él al escucharlo mientras caminamos (SOLNIT, 2015, p. 402s).

\section{Paseos sonoros}

Paseo sonoro es la traducción habitual al español de la expresión soundwalk que empleó Westerkamp en la primera publicación dedicada a esta práctica artística en 1974. Con esa expresión se hace referencia a la conjunción del caminar con la escucha atenta de los lugares y entornos que se van recorriendo. Y si bien Kubisch y Cardiff denominen respectivamente sus trabajos como electrical walks y audio walks, debido a que ellos el sonido es protagonista y la acción consiste en escuchar mientras se recorre por un determinado lugar, entrarían a formar parte del catálogo de lo que se consideran paseos sonoros. No obstante, no se puede obviar que con esos diferentes adjetivos se están apuntando a diferencias que atañen al qué escuchar dentro del amplio espectro que abarca lo sonoro y al cómo hacerlo. Si con el sound, que traducimos por sonoro, parece en principio darse mayor protagonismo a lo que suena, a los sonidos de manera general, el electrical de Kubisch indica que los sonidos que captarán la atención durante el paseo serán eléctricos (esta artista trabaja con campos electromagnéticos). Por su parte, el audio (que viene del verbo latino audire, oír) se decantaría más hacia quien está poniendo la atención, quien escucha mientras está caminando y también recuerda a las audioguías ${ }^{1}$.

Las variedades de paseos sonoros son muchas ya que el caminar escuchando admite muchas variables sean señaladas estas o no en el adjetivo que se emplee. Pueden realizarse en grupo o en solitario, ser más o menos silenciosos, requerir o no de un mapa, estar más o menos meticulosamente trazados, disponer o necesitar de auriculares, micros o cualquier otro dispositivo, apuntar a lo meramente perceptivo o tener implicaciones ecológicas o sociales más o menos explícitas, etc. En todo caso, siempre ponen en juego cuerpos que caminan y escuchan espacios o entornos que son recorridos habitualmente sin que se les preste demasiada atención. Fue Max Neuhaus (1939-2009) quien inauguró la práctica artística del paseo sonoro, aunque

1 Tres años después del texto de presentación de los paseos sonoros de Westerkamp, R. Murray Schaffer en su conocido e importante libro The Soundscape. Our sonic Environment and the Tuning of the World, distinguió entre soundwalk (paseo sonoro) y listening walk (paseo de escucha). Este se concentraría en la escucha, exigiendo un ritmo pausado y tratando de evitar los sonidos que puedan provocar otros participantes (como los sonidos de los pasos ajenos). En el caso del soundwalk, la atención estaría dirigida a la exploración del paisaje sonoro de una zona determinada y se haría uso de mapas e instrucciones que ayuden a atender a sonidos inusuales (SCHAFER, 1994, p. 212-213). Sin embargo, esta distinción no fue seguida y será la amplia definición de Westerkamp la que se imponga de manera generalizada 
sin denominarlo como tal, en $1966^{2}$. En un gesto que recuerda a ese primer paseo dadá del 14 abril 1921 que invitó a salir de las salas de espectáculo para recorrer estéticamente un lugar banal de París (los alrededores de la iglesia de Saint-Julien-lePauvre), este músico propuso a un pequeño grupo de amigos abandonar la habitual sala de conciertos para recorrer parte de Manhattan. Neuhaus estampó sobre las manos de estos paseantes la palabra LISTEN (ESCUCHA) para señalar un tanto imperativamente que estuviesen atentos a todo lo que sonase en esas calles que eran las de su barrio (el paseo concluyó en el propio estudio de Neuhaus donde interpretó algunas piezas para percusión).

Este gesto de salir a la calle a escuchar por parte de Neuhaus fue una respuesta al interés que las músicas de vanguardia o experimentales mostraban por introducir los sonidos de la calle o de nuestro cotidiano en las salas de concierto. Se encuadraba así en el persistente cuestionamiento de los límites entre arte y vida tan ligado a la vanguardia y con todas las problemáticas que esto conlleva. Entre ellas estaría la siempre conflictiva estetización. Sin duda, sacar a los oyentes fuera de los auditorios puede provocar la misma escucha desinteresada y descorporalizada que se produce dentro de esas heterotopías construidas para la escucha y una estetización de cualquier sonido o paisaje sonoro, desvinculando lo que suena de cualquier contexto. Sin embargo, desde el primer momento la práctica del paseo sonoro se reveló una eficaz manera de desprenderse de esa escucha idealizada en favor de otras más situadas y relacionales. De ahí que, si bien hay artistas que prefieren explorar vías centradas en lo exclusivamente sonoro, el efecto que puede tener la realización de paseos sonoros en nuestra relación con los entornos sonoros urbanos y, a partir de ahí, en cómo hacemos ciudad siempre ha estado presente de manera más o menos explícita o latente. Durante un paseo sonoro se nos puede hacer perceptible o podemos tomar conciencia de cómo y a qué solemos prestar atención, qué nos pasa desapercibido, por dónde y cómo solemos caminar, cómo somos parte activa en nuestros entornos sonoros, así como a los afectos, memorias, fantasías que todas estas percepciones pueden generar y a los contextos sociales y ecológicos de nuestros entornos sonoros. De este modo, el paseo sonoro llega a ser una práctica artística que busca no sólo alterar lo que se considera como arte (o música), sino también nuestras vidas mediante la percepción de nuestra implicación en los entornos sonoros.

En esta implicación el propio cuerpo percipiente y caminante adquiere un especial protagonismo cuando se le invita a escuchar en lugar de pretender aparcarlo en el ropero junto con los abrigos. Al salir de las salas de concierto no sólo tendremos oídos para escuchar lo que nos rodea, sino también para esas implacables topías que somos (FOUCAULT, 1988). Porque si desde nuestros cuerpos medimos distancias y cercanías (TUANG, 2001, p. 47), cuando de sonidos y escuchas se trata, esas cercanías se convierten en intimidades resonantes: nuestros cuerpos vibran al escuchar a la vez que se oyen (al caminar, al hablar, al respirar...) formando parte del entorno sonoro. Y en estas mutuas resonancias se ven envueltos los afectos, las experiencias,

2 Después de unos años realizando esos paseos, Neuhaus los bautizó como Lecture Demostrations, el LISTEN estampado como conferencia y el paseo como demostración. 
las memorias, los deseos de esas topías que somos, nunca abstractas ni vacías, nunca ajenas o indiferentes a los lugares vividos, a los espacios cotidianamente recorridos, como muestran las psicogeografías, las geografías emocionales o las topofilias. Por ello, al cambiar el modo de atención, al activar la escucha atenta al caminar, el paseo sonoro puede llegar a alterar cómo nos hacemos cuerpo con nuestros territorios urbanos, cómo los hacemos propios en el sentido que señala Vinciane Despret cuando defiende una apropiación territorial que transforme el espacio no tanto en "suyo" como en "sí" (DESPRET, 2019, p. 36).

\section{Hildergard Westerkamp: salir de nuestras burbujas sonoras.}

En Soundwalking (Paseo sonoro), la primera publicación en la que aparece esta expresión para referirse a la práctica artística del pasear escuchando, Westerkamp definió esta como "cualquier excursión cuyo propósito principal sea escuchar el entorno. Es exponer nuestros oídos a cada sonido que nos rodea, sin importar dónde estemos" (WESTERKAMP, 2019b, p. 111). Estas excursiones no tienen por qué llevarnos muy lejos: se puede escuchar caminando por la calle o por un parque, pero también se puede hacer en casa o en la tienda en la que estemos comprando. Es más, ni siquiera sería necesario caminar, el paseo sonoro puede practicarse mientras se espera de pie o sentada en espacios exteriores o interiores (entre los ejemplos que menciona Westerkamp están la consulta del dentista, la parada del autobús, el aeropuerto...). La excursión fundamental que propone el paseo sonoro sería para esta artista y compositora la que consigue abrir nuestros oídos (directamente) al entorno (WESTERKAMP, 2019b, p. 115).

Westerkamp busca, por tanto, sacarnos de nuestros hábitos de escucha y de las protectoras burbujas acústicas con las que solemos envolvernos para aislarnos de los sonidos que nos rodean cotidianamente en cualquier lugar. Sin embargo, aunque un paseo sonoro se pueda realizar no importa dónde, el que se haga por aquellos espacios públicos que habitualmente recorremos sin prestar demasiada atención multiplica el efecto de esta acción ya que rompe con el hábito de ocupar un espacio interior tras otro haciendo de las calles meros trayectos indiferentes. Es más, tan asumido tenemos este aislamiento acústico que hemos llegado a construir burbujas privadas que hacen de estos trayectos algo interior, algo cerrado al entorno sonoro. Por ello, cuando revise años después este primer texto sobre el paseo sonoro, Westerkamp añadirá a las protectoras paredes y a los cerrados vehículos, el artilugio que probablemente mejor expone el amor que tenemos por esas burbujas acústicas: los auriculares. Tanto es así que incluso los auriculares que se utilizan al grabar el paisaje sonoro, los sonidos que nos rodean, provocan para Westerkamp el efecto burbuja, si bien de un modo que podemos calificar de paradójico ya que a la vez separan y exponen: 
Soundscape recordists exist in their own sound bubble and hear the place in which they are, completely differently from everyone else in the same place. They are like foreigners or outsiders, no matter whether the place is their home or foreign territory. (...) When the recordist is in a foreign country he or she is in fact positioned in a sound bubble within a sound bubble: doubly separated at the same time doubly exposed, naked by virtue of hearing through microphone or headphones and with a stranger's ears (WESTERKAMP, 2010, p. 74).

Por tanto, Westerkamp tiene un particular interés por alterar el modo en el que percibimos nuestros entornos sonoros habituales a través de la práctica del paseo sonoro. Y se trata en su caso de un interés completamente atravesado por las preocupaciones ecológicas que la acucian. Westerkamp quiere que esa alteración en la manera en que escuchamos, ese sacarnos de nuestras burbujas sonoras afecte al modo en el que nos relacionamos con el mundo. No se puede obviar que Westerkamp es una de las cofundadoras del World Soundscape Project, actualmente el World Forum for Acoustic Ecology. Vinculado a la Universidad Simon Fraser de Vancouver, el World Soundscape Project surgió entre finales de los años sesenta y los setenta como un grupo educativo y de investigación del que también formaban parte R. Murray Schafer, Bruce Davis, Peter Huse, Barry Truax, Howard Broomfield. Preocupados por el entorno sonoro y el problema de la contaminación acústica así como por el rápido cambio de los paisajes sonoros (soundscapes, término acuñado por Schafer), los miembros del World Soundscape Project salieron con sus equipos de grabación de audio para realizar unas investigaciones de campo que compartirían en piezas sonoras, artículos, libros, conferencias y programas de radio (como la serie Soundscape of Canada, cuyos diez programas fueron emitidos por la CBC entre el 21 de octubre y el 1 de noviembre de 1974) ${ }^{3}$.

En buena medida, los paseos sonoros permiten a cualquiera poder experimentar y adentrarse en las cuestiones que preocupaban a los integrantes del World Soundscape Project: escuchar el entorno sonoro, atender a lo que nos agrada y a lo que nos desagrada del mismo, a lo que nos llama la atención, a lo que hasta ese momento nos ha pasado desapercibido, etc. Y todo ello sin necesidad de ir con un equipo de grabación (aunque sin excluirlo tampoco), tan sólo abriendo los oídos para tratar de "activar" una escucha atenta. Además, si se practica con asiduidad, el paseo sonoro permite prestar atención a los cambios que se van produciendo en unos paisajes sonoros que no sólo es que nos envuelvan, sino que formamos parte activa de ellos. De ahí la importancia que para Westerkamp tiene la posibilidad de que se vaya más allá del deleite estético para buscar una respuesta activa por parte de quien pasea escuchando que le lleve a actuar y a desarrollar entornos acústicos de buena calidad. E insiste en la especial pertinencia de que esto se produzca en las mismas ciudades

3 Más información sobre el World Soundscape Project se puede encontrar en https://www.sfu. ca/ truax/wsp.html. 
en las que vivimos, no sólo cuando salimos al campo a pasear, pues están llenas de pistas acústicas que son importantes para nuestra supervivencia (algo que cada vez se está haciendo más evidente gracias a las investigaciones acerca de los nocivos efectos de la contaminación acústica para quienes vivimos, humanos y no humanos, en las ciudades) ${ }^{4}$. Al permitirnos percibir nuestras propias acciones acústicas, los paseos sonoros nos ayudan a ser conscientes de nuestra responsabilidad colectiva con el entorno sonoro del que formamos parte, a percatarnos y a responder tanto a la contaminación acústica como a la desaparición de sonidos y paisajes sonoros: "A menos que escuchemos con atención, se corre el riesgo de que algunos de los sonidos más delicados y silenciosos pasen desapercibidos entre los oídos insensibilizados y entre las muchas voces mecanizadas de los paisajes sonoros modernos, y de que finalmente puedan desaparecer por completo" (WESTERKAMP, 2019b, p. 112). Westerkamp está convencida de que la práctica continuada del paseo sonoro lleva a la exigencia de una mejor calidad de los entornos sonoros urbanos y a tomar decisiones que, aunque simples, tienen consecuencias (entre los ejemplos que menciona está el no poner la radio todo el día, el utilizar maquinaria silenciosa, ayudar a preservar las zonas tranquilas de nuestras ciudades, etc.) (WESTERKAMP, 2019b, p. 118). Así pues, ni quien escucha ni lo escuchado saldrían indemnes de estos paseos sonoros.

Ese atender a los sonidos que producimos que forman del paisaje sonoro en el que estamos inmersos incluye los sonidos de nuestro propio cuerpo al caminar. Es más, el que podamos escuchar estos sonidos sería para Westerkamp un claro indicador de la calidad del entorno sonoro: "Si puedes escuchar hasta el más silencioso de estos sonidos significa que atraviesas un entorno dimensionado a escala humana. En otras palabras, con tu voz o tus pasos, por ejemplo, 'hablas' con tu entorno, que a su vez responde aportando a tus sonidos una cualidad acústica específica” (WESTERKAMP, 2019b, p. 113). Por ello, Westerkamp propone arrancar los paseos sonoros escuchando el propio cuerpo en movimiento de quien pasea escuchando. Ahora bien, no se trataría de centrarse únicamente en él, sino de tomar conciencia de que forma parte del entorno ya que desde esa autopercepción se pueden sentir los sonidos más cercanos y los más lejanos, y hasta componer un paisaje con ellos. De este modo, se podrían en práctica en el ámbito de lo sonoro ese medir distancias y cercanías desde el propio cuerpo del que hablan Tuang y Foucault para trazar un territorio que se conoce y ocupa escuchando y caminando a la vez que se conoce el propio cuerpo. Nos compondríamos y nos apropiaríamos con él, como diría Despret.

4 Una muestra de lo importante que es para Westerkamp el pararse a escuchar y recorrer las ciudades en las que vivimos es la serie de paseo sonoros Sounwalking que realizó entre 1978 y 1979. Fue emitida por Co-op Radio, una estación de radio comunitaria no comercial de Vancouver que empezó a emitir en 1974 y sigue activa (http://www.coopradio.org/). En ella se pudieron escuchar paseos a través del centro comercial, de parques, del zoo, de una fábrica, de áreas residenciales que están bajo las rutas de los aviones, de las calles de Vancouver, etc. Para Westerkamp este trabajo radiofónico también es importante porque la comparación entre cómo escuchan nuestros oídos y cómo capta el micrófono los sonidos ayuda a activar nuestra escucha, a darnos cuenta de aquello a lo que no prestamos atención, a lo que nos pasa desapercibido o consideramos poco importante. Además, y no menos importante, con estos paseos sonoros emitidos por radio se potenciaba, se evidenciaba la narrativa que un paseo sonoro encierra. 
Es importante no dejar de señalar que el acento que pone Westerkamp en el paseo sonoro como práctica ecológica no pone en cuestión que se trate ante todo de una práctica artística. En esto, esta artista se muestra próxima al sentido de ecología defendido por Félix Guattari y su apuesta por generar desde el arte y cualquier actividad creativa otros modos de percibir que alteren subjetividades y relaciones con los demás y con una naturaleza de la que somos parte, como lo son nuestras ciudades. En una conferencia pronunciada en 2006 titulada "Soundwalking as Ecological Practice", Westerkamp afirmó que "in a soundwalk then, listeners and the environment create a unique 'piece' together" (WESTERKAMP, 2006). Esta conferencia y estas palabras son una clara evidencia de que, con el paso del tiempo, sigue convencida de que esta práctica es una preciosa manera de activar una escucha que nos hace percibir, experimentar que somos parte del entorno en el que habitamos. Durante tantos años de práctica ha podido comprobar cómo, invariablemente e independientemente de la experiencia como oyente-caminante, los efectos de los paseos sonoros son inmediatos y afectan tanto a la apertura de los oídos y la activación de la escucha, como también a los demás sentidos. Y es más, "not only does a soundwalk raise general consciousness towards the acoustic environment, it also creates a living connection between listener and place" (WESTERKAMP, 2006). Esta conexión viva atañe al cuerpo percipiente y caminante con el lugar que percibe y camina a la vez que se percibe al hacerlo, que se apropia del espacio haciéndose parte de él: "a unique piece together". Asimismo, no se olvida Westerkamp de mencionar que en este apropiarse, siempre impredecible, también entran en juego experiencias, memorias, afectos, deseos y temores vinculados a los lugares que se recorren. De ahí que califique al paseo sonoro de trabajo situacional haciendo referencia a la física y feminista Ursula Franklin y que afirme que "a soundwalk does not only reveal relationships within the acoustic environment but perhaps more importantly, makes relationships conscious between listeners' experiences and their acoustic - social environment" (WESTERKAMP, 2006).

Ese impredecible señalado por Westerkamp apunta a un elemento clave en el paseo sonoro que atañe a la disponibilidad de quien participa y que va a afectar a subjetividades y relaciones: la temporalidad que se pone en juego cuando se camina escuchando o se escucha caminando. Este aspecto lo explicitará la propia Westerkamp en otra conferencia titulada "The Disruptive Nature of Listening" y pronunciada en 2015. Para que ese impredecible pueda tener lugar es necesario querer abrir los oídos, disponerse exponiéndose hacia lo que suena sin importar las reacciones que esto pueda provocarnos: sorpresa, incomodidad, molestia, dificultad, placer ${ }^{5}$. Sin embargo, esta disposición requiere algo nada fácil para una vida cotidiana empujada por el continuo 24/7 asumido ya casi de manera inconsciente: parar. Cuando lo habitual es sentir como un tiempo perdido, como unobstáculo a salvar lo más rápidamente posible el tener que desplazarse a pie de un lugar a otro, pasear lentamente para escuchar las calles que

5 En "Paseo sonoro", Westerkamp habla de "escucha sin compromiso", con la que esbozaba el tipo de disposición que años después desarrollará con algo más de detalle en "The Disruptive Nature of Listening" (WESTERKAMP, 2019b, p. 112). 
habitualmente recorremos puede llegar a ser un gesto tremendamente perturbador: "The decision to do it, rather than talk about it, is the first disruption of old patters. The experience of soundwalk listening then gives us a visceral understanding of what in fact the disruptive nature of listening really is" (WESTERKAMP, 2019a, p. 24). Para Westerkamp la escucha es intrínsecamente disruptiva porque logra romper los flujos habituales del tiempo, nos saca del comportamiento habitual de una vida cotidiana cada vez más extenuada y extenuante. De ahí que convertir la escucha y el paseo sonoro en una práctica continua, tratar de situarse en un estado de atención continuo provoca inevitablemente cambios en quien escucha, en su relación con el tiempo, en su relación con los demás, en el paisaje sonoro. Por todo ello, Westerkamp está convencida de que esta disposición que la escucha ofrece y el paseo sonoro pone en práctica nos puede ayudar a tratar con los desafíos ambientales, sociales y políticos a los que nos estamos enfrentando.

\section{Christina Kubisch: nada se ve como suena y nada suena como se ve}

Entre la multitud de sonidos que pueblan nuestros entornos urbanos y que nos pasan desapercibidos se hayan no pocos que escapan a lo que los oídos humanos más atentos son capaces de captar. Es el caso de la paleta sonora con la que trabaja Kubisch, quien desde finales de la década de 1970 investiga cómo hacernos oír los campos electromagnéticos que nos envuelven en nuestro día a día. En 2004, en la ciudad alemana de Colonia, Kubisch decidió sacar a la calle estas investigaciones para que pudiésemos escuchar la enorme variedad de sonidos que producen estos campos generados por los diferentes dispositivos eléctricos y electrónicos que encontramos por doquier en nuestras ciudades: wifi, cajeros automáticos, sistemas de iluminación, sistemas de comunicación inalámbrica, sistemas de radar, dispositivos de seguridad antirrobo, cámaras de vigilancia, teléfonos móviles, ordenadores, antenas, sistemas de navegación, redes de transporte público, etc. Así nacieron sus Electrical walk, de los que ha realizado más de 6o entre 2004 y 2017 por toda Europa y América del Norte, además de Hongkong, Lagos y Bangkok ${ }^{6}$.

Imprescindible para realizar estos paseos eléctricos es el empleo de unos auriculares construidos por la propia Kubisch con bobinas integradas que son capaces de captar y amplificar las cualidades acústicas de los campos electromagnéticos. Si retomamos la idea de Westerkamp de las burbujas sonoras, podemos afirmar que estos particulares auriculares separarían a quien los porta del resto de personas con las que comparte el espacio para adentrarse en una ciudad en la que lo que se ve y lo que se oye no coinciden: "Nothing looks the way it sounds. And nothing sounds the way it looks" (KUBISCH, 2017). Kubisch nos hace perceptible una ciudad inaudita e invisible que, aunque presente, nos es habitualmente inaccesible por más que

6 En este enlace se puede ver en detalle los paseos eléctricos que ha realizado Kubisch http://www. christinakubisch.de/en/works/electrical_walks. Y en este otro se puede escuchar una muestra de los sonidos que ha ido recopilando en ellos http://www.cabinetmagazine.org/issues/21/kubisch.php 
afecte a nuestra vida (e incluso a nuestra salud). Aunque los sonidos los produzcan dispositivos no están para nada ocultos, podemos hablar de ciudad invisible por esa fuerte discrepancia que se produce entre lo que se ve y lo que se oye (COX, 2006; PARDO SALGADO, 2015, p. 25).

La inmersión en este universo sonoro desconocido a la vez que cotidiano altera la percepción de la realidad cotidiana ya que esta aparece en un nuevo contexto en el que cobra protagonismo lo que no somos capaces de percibir, lo que nos pasa desapercibido. Son las calles de siempre, pero parecen otras al llenarse de presencias invisibles. También nuestra forma de movernos cambia. A Kubisch le gusta observar cómo se mueven quienes participan en unos paseos en los que no se escucha lo mismo dependiendo de cómo nos movamos: cada movimiento de la cabeza produce grandes cambios en la manera y la amplitud del sonido que se escucha a través de los auriculares. Hay quien se detiene a escuchar, hay quien corre de un lado para otro para generar determinados juegos de sonidos. Estas coreografías improvisadas, sin menospreciar su lado lúdico, muestran la importancia que tiene la manera de movernos en el espacio público, en las calles, al sacar a relucir nuestros hábitos al caminar (qué consideramos apropiado, ante qué nos solemos detener o dónde aceleramos el paso). Aunque, sobretodo, ponen en de manifiesto el profundo vínculo que se da entre cómo percibimos la ciudad y cómo nos movemos en ella. Los especiales auriculares de Kubisch continúan y exploran así la vía abierta por esos auriculares más corrientes que empezaron a conectarse a los walkman a principios de la década de los ochenta y que han terminado formando parte importante en nuestro cotidiano recorrer calles, parques y transportes públicos acompañados por una banda sonora particular. No podemos olvidar que desde el primer momento de explosión del uso de los walkman se destacó cómo se vio afectada la manera de caminar por la ciudad:

Geared with headphones, the walkman listener strolls along and takes in the musical scenery on his/her way. A kind of tuning in is created between his ear and his step. New sonic territories are composed in the course of this mobile listening experience. As the body moves in sync to the music, the listener transforms the public scene and provides a new tonality to the city street. His footsteps seem to say what his ears may be trying to hide (THIBAUD, 2003, p. 329) ${ }^{7}$.

Las complejas capas de frecuencias altas y bajas, bucles de secuencias rítmicas, grupos de señales diminutas, drones largos y muchos otros sonidos indescriptibles que generan los campos electromagnéticos hacen de la ciudad misma un continuo concierto secreto al cual se puede acceder, y que se puede bailar, con ayuda de los auriculares y de los mapas que ofrece Kubisch. En ellos, la artista indica posibles

7 Sobre el efecto del walkman en nuestra manera de movernos y caminar por la ciudad véase también HOSOKAWA, 1984 . 
rutas o elementos especialmente interesantes que se pueden recorrer o visitar de manera individual o en grupo. Desde esta idea de la ciudad como concierto, estos mapas operarían a modo de partituras que seguimos con nuestros cuerpos (MILANI, 2009).

Todos estos años recorriendo el mundo con los paseos eléctricos le ha permitido ir dibujando un mapa de mayores dimensiones en el que ha ido recopilando los sonidos y sus lugares, un mapa general en continua expansión, no sólo por la actividad de Kubisch, sino por la cada vez mayor presencia de dispositivos e instalaciones que generan campos electromagnéticos. Algunos sonidos son muy parecidos en todo el mundo, respondiendo a la globalización del sonido. Otros son específicos para una ciudad o país y no se pueden encontrar en ningún otro lugar. En relación a este mapa global y la expansión de los dispositivos que generan campos electromagnéticos, la propia artista reconoce que estos paseos sonoros eléctricos no sólo son un trabajo artístico, sino también una especie de investigación social (por ejemplo, permiten conocer la distribución de los dispositivos e instalaciones en nuestro entorno con sus implicaciones económicas, sociales, ecológicas o políticas). Ahora bien, también afirma que, aunque siempre ha sido crítica con la forma en que nos manejamos con la tecnología, en su trabajo no trata de señalar qué es bueno o qué es malo, sino que lo que le interesa es que la gente reconozca lo que les rodea por sí misma. Haciendo audible parte de eso inaudible que producimos y con lo que convivimos en nuestras ciudades, Kubisch no trata sólo de ampliar nuestro catálogo de sonidos. Al igual que Westerkamp, con sus paseos Kubisch busca alterar nuestra percepción, que prestemos atención a lo que nos pasa desapercibido y con ello a nuestra relación con nuestros entornos sonoros urbanos (MOTTE-HABER, 2009, p. 22; PARDO SALGADO, 2016a, p. 92).

A ello se añade un acercamiento a la pregunta por cómo nos manejamos con la tecnología que no puede ser maniquea o simplista ya que Kubisch nos coloca con sus paseos eléctricos ante parte de la complejidad que entraña nuestra relación inevitable con omnipresentes dispositivos tecnológicos de muy distinto carácter. No sólo se trata de lo bellos que nos puedan parecernos los sonidos generados por dispositivos que consideremos más o menos cuestionables o perniciosos, o que nos asalte la cuestión de cuánta contaminación acústica a la que los humanos somos sordos pueda afectar a otros seres vivos o a nosotros mismos sin percatarnos ${ }^{8}$. Porque sin los auriculares diseñados por la propia Kubisch ni siquiera seríamos capaces de oír esa ciudad electromagnética invisible en la que vivimos. Le cuestión es qué, cómo y para qué hacemos con la tecnología de la disponemos o que podemos crear. Y si bien Kubisch no dice qué es bueno o malo, qué o cómo deberíamos de hacer, lo cierto es que ella nos ofrece un dispositivo que nos permite oír lo imperceptible fomentando así otras maneras de percibir y, con ellas, de relacionarnos y de habitar e imaginar

8 Un acercamiento a los sonidos inaudibles a los oídos humanos pero bien presentes para otros seres vivos es el que hace la artista Jana Winderen en su instalación de 2017 Rats - Secret Soundscapes of the City. En ella nos permite oír a los humanos esos paisajes sonoros a los que somos sordos y en los que están inmersos las ratas y otros seres con oídos sensibles a ultrasonidos. 
nuestras ciudades mucho más atentas a todo lo que se nos escapa y puede estar cargado de belleza, interés o, quizá, peligro.

\section{Janet Cardiff: laberintos temporales}

Los auriculares también son imprescindibles en los audio walks ideados por Cardiff, si bien en su caso son auriculares corrientes conectados a algún dispositivo de reproducción de audio de los que habitualmente utilizamos. Sus paseos, por tanto, comienzan con un gesto que se ha convertido en cotidiano en nuestras vidas urbanas: colocarse unos auriculares, darle al play y caminar por la calle -o por el parque, o por el museo - . A través de esos auriculares se oye a la propia artista dando las indicaciones necesarias para realizar el paseo al modo de las audioguías, por lo que no es tan extraño que Cardiff se decante por llamarlos audio walks, en lugar de soundwalks. Lo primero que pide esa voz es acompasar los pasos que se van a dar con los pasos que se pueden oír a través de esos auriculares (que son también los de la artista). Este acompasarse no es para nada anecdótico o banal. Arriba hemos mencionado cómo en su primer escrito sobre paseos sonoros Westerkamp había señalado la importancia de poder escuchar los propios pasos no sólo como indicador del nivel de contaminación acústica del entorno, sino como una sencilla forma de componerse con el paisaje sonoro del que se es parte. Unos años después, Cardiff hará de los pasos que suenan al caminar un elemento compositivo clave en sus audio walks puesto que con ellos logrará introducirnos en un entorno sonoro urbano compartido y compuesto siempre con otros y otras.

Cardiff comenzó de manera azarosa a realizar este tipo de paseos sonoros en 1991: mientras paseaba por un bosque de Canadá grabando con un walkman notas de audio, se equivocó en un momento dado de botón y pudo escucharse a sí misma hablando en el mismo lugar (paisaje sonoro) en el que se encontraba, pero en un momento distinto. Cardiff experimentó entonces cómo sus oídos se ponían en alerta tratando de distinguir lo grabado de lo real, así como la sugerente capacidad narrativa que poseía este inesperado pliegue temporal. De este modo, la habitual burbuja ofrecida por los auriculares vuelve a trastocarse: expone a quien escucha a lo que está sonando a su alrededor a la vez que le aísla al sumergirle en una historia que alterará su relación con el lugar que está recorriendo y con la ciudad en la que habita.

Esta exposición perceptiva simultánea a una inmersión narrativa se ve favorecida por la técnica de grabación binaural empleada por Cardiff. Mediante ésta se trata de reproducir el espacio sonoro tridimensional captado por los oídos humanos gracias a dos micrófonos omnidireccionales colocados en las orejas de quien graba a modo de unos auriculares (de nuevo nos volvemos a encontrar con unos auriculares que aíslan y exponen al mismo tiempo). Con estos micrófonos binaurales Cardiff (que, en lugar de sus propias orejas, emplea las de una cabeza de maniquí) registra el mismo recorrido que hará posteriormente quien siga su audio 
paseo. De este modo, al hacer el paseo se escuchan los sonidos que se están dando en ese momento en las calles, parques o edificios a la vez que aquellos que se dieron cuando Cardiff los grabó sin poder distinguirlos entre sí con certeza. Por ello, es prácticamente inevitable volverse para comprobar si los pasos que sentimos a nuestra espalda los está dando alguien que nos sigue o que siguió en su momento a quien grabó el recorrido. A Cardiff también le gusta jugar con sorprendentes encuentros (o desencuentros), como cuando el músico callejero que anuncia la voz guía está tras la esquina interpretando la misma música que la artista escuchó y registró unos meses antes.

Mediante estas confusiones sensoriales Cardiff logra que, por más inmersos que estemos en lo que nos va contando, nuestros sentidos no dejen de estar alerta a lo que nos rodea. Porque el relato en el que nos sumerge va mucho más allá de dar indicaciones sobre qué esquina doblar o a qué sonido prestar atención sin perder el compás de sus pasos. Para cada uno de sus paseos urde un relato que no sólo alentará nuestro interés por acabar el paseo, sino que nos trama de maneras inesperadas con nuestros espacios habituales: "The virtual recorded soundscape has to mimic the real physical one in order to create a new world as a seamless combination of the two. My voice gives directions but also relates thoughts and narrative elements which instill in the listener a desire to continue and finish the walk" (SCHAUD, 2005, p. 91). De nuevo, algo apuntado por Westerkamp, que al pasear escuchando se cuentan historias, Cardiff lo va a explorar de manera radical en su trabajo: con sus paseos sonoros nos va a ir llevando por calles, parques y otros espacios públicos siguiendo sonidos, palabras, memorias, deseos, temores e incluso la historia que hacen de la ciudad un lugar vivido y vivo, y con los que construye sus relatos (el verbo to relate conjugaría a la perfección ese relacionar narrando). Si los paseos de Kubisch nos hacen perceptibles esos campos electromagnéticos con los que convivimos continuamente, los de Cardiff nos permiten escuchar e implicarnos en la multitud de relaciones, pliegues y capas invisibles que construyen y relatan la ciudad en la que vivimos. No sólo de asfalto, ladrillo o piedra están hechas las ciudades.

Entre esos pliegues están los que provoca un vagar por el tiempo que, si bien es propiciado por el caminar (SOLNIT, 2015, p. 23), en el trabajo de Cardiff alcanza altas cotas de complejidad y sutileza. Pasear con esta artista es introducirse en un laberinto temporal cuyos pliegues y cruces conducen a diferentes momentos vividos o imaginados en las mismas calles, las mismas plazas, los mismos lugares en los que se está. En algunos de sus paseos llegar a hacer explícitos estos juegos temporales. Por ejemplo, durante Münster Walk se escucha: "You are walking in the present, listening to a recording of my voice from at least two months before, which is describing a memory from a past event. Where in time is that memory for the listener?" (SCHAUD, 2005, p. 113). A través de estos pliegues y encuentros temporales, el microcosmos personal de quien la sigue durante uno de sus paseos se va vinculando con otros microcosmos no menos íntimos, así como con macrocosmos públicos. Además, Cardiff consigue ir enlazando espacios interiores y exteriores, mucho más porosos entre sí de lo que solemos creer. Estos espacios interiores no son sólo evocados o 
mencionados, ya que pueden delatarse por sus características acústicas. Es decir, Cardiff también registra en espacios cerrados como iglesias y teatros para llevarnos hasta dentro de ellos siguiendo un recuerdo, una conversación o incluso una música sin tener que pisarlos. El sonido puede llevarnos a muy diferentes lugares, muchas veces inesperados, sin movernos de donde estemos, sólo hay que escuchar:

I think sound has the ability to transport you to a different place. It takes you away from your current reality into another room. Say you listen the radio. Then you hear seagulls and all of a sudden you feel like you are on the beach. Sound is so special for me. it's a very physical thing. And I enjoy the idea of building this virtual and invisible world around us, how it's there, but not really there. A lot of times I think that sound enables us to escape from the reality we are in (CARDIFF e MILLER, 2014, p. 129).

En esos espacios a los que puede llevar estarían no sólo los espacios grabados, evocados o mencionados por Cardiff, sino también los que recuerden o imaginen por quienes están paseando y escuchando.

Por otra parte, en estas tramas espacio-temporales que son los paseos sonoros de Cardiff, lo inesperado y el misterio juegan un papel importante. Sin duda ambos son un buen aliciente para mantener la atención hasta el final del paseo. Her Long Black Hair (2004), paseo por Central Park siguiendo el rastro de unas fotografías de una mujer en ese mismo parque, y The telephone call (2001), paseo en el que nos confundimos con una mujer que siente que la están siguiendo, son una buena muestra de ello (por cierto, en ambos están presentes los peligros y temores asociados a una mujer caminando sola por la ciudad, por un parque o, incluso, por un museo ya que el segundo de los paseos señalados tiene lugar dentro del Museo de Arte Moderno de San Francisco). Sin embargo, no se trata sólo intrigar para entretener. Por ejemplo, sobre The telephone call, que estrictamente hablando es un video walk (se hace también uso del vídeo), Cardiff afirma que "the basis of the narrative was about how our minds invent scenarios from chance meetings between people. The piece was largely about self-induced anxieties and how the fears we have change our perception of our world" (SCHAUD, 2005, p. 292). Por tanto, con el elemento de misterio busca evidenciar cuánto afecta lo que sentimos tanto a lo que percibimos como a nuestras relaciones con los demás y con los distintos espacios públicos.

Así pues, Cardiff también quiere alterar el modo en el que percibimos y nos relacionamos mediante la práctica del paseo sonoro. En su caso, tiene especial interés en que prestemos tiempo y atención a las ciudades en las que vivimos como lugares compartidos y llenos de afectos, memorias, deseos, historia y posibilidades. Se trata de no olvidar los pasados colectivos y personales estando situadas y situados en el presente escuchando atentamente y abiertos a los inesperados encuentros que puedan darse a la vuelta de cualquier esquina: "Walking is very calming. One step after another, one foot moving into the future and one in the past. Did you ever think 
about that? It's like our bodies are caught in the middle. The hard part is staying in the present. Really being here" (CARDIFF, 2004).

\section{Conclusión}

Westerkamp, Kubich y Cardiff proponen paseos sonoros bien diferentes atendiendo a qué escuchar y la manera de hacerlo mientras se pasea por lugares que frecuentamos en nuestro día a día, principalmente las calles de las ciudades en las que vivimos. Westerkamp nos invita a salir de nuestras habituales burbujas sonoras protectoras para tomarnos el tiempo de escuchar los sonidos que componen unos entornos urbanos sonoros de los que formamos parte activa y, por tanto, somos responsables. Kubisch nos introduce en una ciudad invisible cuyo paisaje sonoro está compuesto por dispositivos e instalaciones eléctricos y electrónicos a los que somos sordos y de los que nos hemos hecho dependientes y que cada vez son más numerosos. Y, por su parte, Cardiff nos relata y trama con las muchas y diferentes ciudades vividas dentro de una misma ciudad siguiendo sonidos, afectos, memorias y voces propias y ajenas. Sin embargo, tan diferentes propuestas comparten algo más que el que se trate de la misma práctica artística. Estas tres artistas nos invitan a escuchar mientras paseamos las ciudades en las que vivimos con la intención de que percibamos la enorme riqueza sonora que nos rodea y atraviesa, cuánto nos pasa desapercibido por no prestar atención, por no escuchar a pesar de ser activos partícipes de aquello a lo que estamos siendo sordas y sordos. Las tres, por tanto, nos llevan a descubrir mediante el sonido otras ciudades dentro de nuestra misma ciudad alterando así el modo en que las percibimos, las escuchamos. Y las tres buscan que con esta alteración de la percepción algo cambie en la manera en la que habitamos y hacemos ciudad, cada una con sus distintos focos o enfoques: de la ecología de Westerkamp, a la relación con la tecnología y lo imperceptible de Kubisch o a la historia y la ciudad compartida en el caso de Cardiff. Estas tres artistas nos muestran, por tanto, que nuestras ciudades son más complejas de lo que creemos, que el sonido es parte fundamental en ellas y de quienes las habitan, y que al pasearlas escuchando con atención en tanto que cuerpos percipientes y afectivos cargados de experiencias, memorias, anhelos, temores, vínculos inevitablemente nos componemos con ellas, algo que nos afecta y afecta a cómo (nos) hacemos ciudad.

\section{Referencias}

CARDIFF, Janet, Her Long Black Hair, 2004.

CARDIFF, Janet, MILLER, George Bures. Something Strange This Way. Ostfilden: Gerd Hatje Cantz, 2014. 
CARERI, Francesco. Walkscapes. El andar como práctica estética. Barcelona: Gustavo Gili, 2005 .

COX, Christoph. Invisible Cities: An Interview with Christina Kubisch. Cabinet magazine, Issue 21, 2006. http://cabinetmagazine.org/issues/21/cox.php Accessed 03/10/2020.

DESPRET, Vinciane. Habiter en oisseau. Arles: Actes Sud, 2019.

FOUCAULT, Michel. Topologías. Dos conferencias radiofónicas. Fractal. Revista Trimestral, n. 48, 1988, p. 48-54.

GUATTARI, Félix. Las tres ecologías. Valencia: Pre-Textos, 2000.

GUATTARI, Félix, ROLNIK, Suely. Micropolítica / Cartografías del deseo. Madrid: Traficantes de sueños, 2006.

HOSOKAWA, Shuhei. The Walkman Effect. Popular Music, 4, 1984, p. 165-18o.

KUBISCH, Christina. Electrical Walks. Electromagnetic Investigations in the City. 2017. In: http://www.christinakubisch.de/en/works/electrical_walks Accessed: $3 / 10 / 2020$

MILANI, Mateo. Walking In The City With Christina Kubisch, Digimad, 45, 2009. In: http://digicult.it/digimag/issue-045/walking-in-the-city-with-christina-kubisch/ Accessed: 3/10/2020

MOTTE-HABER, Helga de la. Concepciones del arte sonoro. Ramona revista de artes visuales, n. 96, 2009, p. 20-25.

NEUHAUS, Max. Listen. In: LANDER, Dan, LEXIER, Micah (ed.). Sound by Artists. Toronto: Art Metropole, 1990, p. 63-67.

PARDO SALGADO, Carmen. Cartographies of Simultaneity. International Journal of Art and Art History, n. 2, vol. 3, 2015, p. 17-29.

PARDO SALGADO, Carmen. Klangkunst y Sound Art: reflexiones y consecuencias. En: FONTÁN DEL JUNCO, Manuel, IGES, José, MAIRE, José Luis (eds.). Escuchar con los ojos. Arte sonoro en España (1961-2016). Madrid: Fundación Juan March, 2016a, p. 86-97.

PARDO SALGADO, Carmen. Une musique pour habiter le monde. En: SOLOMOS, Makis, BARBANTI, Roberto, LOIZILLON, Guillaume, PAPARRIGOPOULOS, Kostas, 
PARDO, Carmen (editores). Musique et écologies du son. París: L'Harmattan, 2016b, p. 197-208.

SCHAFER, R. Murray. The Soundscape. Our sonic Environment and the Tuning of the World. Rochester: Destiny Books, 1994.

SCHAUD, Mirjam (ed.). Janet Cardiff: The Walk Book. Viena: Thyssen-Bornemisza Art Contemporary, 2005.

SOLNIT, Rebecca. Wanderlust. Una historia del caminar. Madrid: Capitán Swing, 2015.

THIBAUD, Jean-Paul. The sonic composition of the city. In: BULL Michael \& Les Back (Eds.). The Auditory Culture Reader. Amsterdam: Berg Publishers, 2003, p. 329-341

TUANG, Yi-Fu. Space and place. The perspective of Experience. Minneapolis: University Minnesota Press, 2001.

WESTERKAMP, Hildegard. Soundwalking as Ecological Practice. In: The West Meets the East, Proceedings for the International Conference on Acoustic Ecology. Hirosaki University,Hirosaki, Japan. November 2-4, 2006. https://www.hildegardwesterkamp. $\mathrm{ca} /$ writings $/$ writingsby $/$ ?post_id $=14 \&$ title $=\% \mathrm{E} 2 \% 8 \mathrm{o} \% 8 \mathrm{~B}$ soundwalking-as ecological-practice- Accessed: 3/10/2020

WESTERKAMP, Hildegard. Speaking from inside the soundscape. Zehar, n. 67, 2010, p. 66-79.

WESTERKAMP, Hildegard. La nature perturbatrice (disruptive) de l'écoute. Filigrane. Musique, esthétique, sciences, société, Usages du son et écoute, L'éthique de la musique et $d u$ son, 2019a, URL: https://revues.mshparisnord.fr:443/filigrane/index. php?id=86o Accessed: 3/10/2020

WESTERKAMP, Hildegard. Paseo Sonoro. En: ESPEJO, José Luis (ed.). Escucha por favor. Madrid: Exit Publicaciones, 2019b, p. 111-126.

Recebido em 30/10/2020.

Aceito em 23/11/2020. 